\title{
Nomogram for predicting cancer specific survival in inflammatory breast carcinoma: a SEER population-based study
}

\author{
Haige Zhang ${ }^{\text {Equal first author, } 1,2}$, Guifen Ma ${ }^{\text {Equal first author, } 1}{ }^{\text {, Shisuo Du }}{ }^{1}$, Jing Sun ${ }^{1}$, Qian Zhang ${ }^{1}$, Baoying Yuan ${ }^{1}$, Genwen \\ Chen $^{3}$, Zhaochong Zeng \\ Xiaoyong Luo \\ resp. 2 \\ ${ }^{1}$ Department of Radiation Oncology, Zhongshan Hospital, Fudan University, Shanghai, China \\ 2 Department of Radiation Oncology, Luoyang Central Hospital affiliated to Zhengzhou University, Luoyang, China \\ 3 Radiation Oncology, Zhongshan Hospital Fudan University, Shanghai, China \\ Corresponding Authors: Zhaochong Zeng, Xiaoyong Luo \\ Email address: zeng.zhaochong@qq.com, luoxiaoyongly@sina.com
}

The clinicopathological features of inflammatory breast carcinoma (IBC), the effect of therapeutic options on survival outcome and the identification of prognostic factors were investigated in this study. Information on IBC patients were extracted from the Surveillance, Epidemiology, and End Results (SEER) database between 2010 and 2015. Cox proportional hazard regression was used to determine potential significant prognostic factors of IBC. A nomogram was then constructed to evaluate patient survival based on certain variables. Univariate and multivariate analyses revealed that race $(p<0.001), M$ stage $(p<0.001)$, surgery $(p=0.010)$, chemotherapy (CT) $(p<0.001)$, tumor size $(p=$ $0.010)$, estrogen receptor $(p<0.001)$, progesterone receptor $(p=0.04)$, and human epidermal growth factor receptor $2(p<0.001)$ were all independent risk factors. The concordance index ( $C$-index) of the nomogram was 0.735 , which showed good predictive efficiency. Survival analysis indicated that IBC patients without CT had poorer survival than those with CT $(p<0.001)$. Stratified analyses showed that modified radical mastectomy (MRM) had significant survival advantages over non-MRM in patients with stage IV IBC ( $p=$ 0.031). Patients treated with or without CT stratified by stage III and stage IV showed better survival than those without stage III and IV $(p<0.001)$. Trimodality therapy resulted in better survival than surgery combined with CT or CT alone $(p<0.001)$. Competing risk analysis also showed the same results. The nomogram was effectively applied to predict the 1, 3 and 5 -year survival of IBC. Our nomogram showed relatively good accuracy with a C-index of 0.735 and is a visualized individually predictive tool for prognosis. Treatment strategy greatly affected the survival of patients. Trimodality therapy was the preferable therapeutic strategy for IBC. Further prospective studies are needed to validate these findings. 
1 Nomogram for predicting cancer specific survival in inflammatory breast carcinoma: a

\section{SEER population-based study}

3 Haige Zhang ${ }^{1,2 \#}$, Guifen $\mathrm{Ma}^{2 \#}$, Shisuo $\mathrm{Du}^{2}$, Jing Sun², Qian Zhang ${ }^{2}$, BaoYing Yuan ${ }^{2}$, Xiaoyong

$4 \mathrm{LuO}^{1}$

$5{ }^{1}$ Department of Radiation Oncology, Zhongshan Hospital, Fudan University, Shanghai 200032 ,

6 China;

$7 \quad{ }^{2}$ Department of Radiation Oncology, Luoyang Central Hospital affiliated to Zhengzhou

8 University, Luoyang, Henan 471000, China;

9

10 \#These authors contributed equally to this work.

11 Corresponding author:

12 Xiaoyong Luo, Department of Radiation Oncology, Luoyang Central Hospital affiliated to

13 Zhengzhou University, 288 Zhong Zhou Zhong Road, Luoyang, Henan, 471000, China,

14 Phone: +86 18537919369

15 Fax:+86(0379)63892244

16 Abstract 
17 The clinicopathological features of inflammatory breast carcinoma (IBC), the effect of therapeutic options on survival outcome and the identification of prognostic factors were investigated in this study. Information on IBC patients were extracted from the Surveillance, Epidemiology, and End Results (SEER) database between 2010 and 2015. Cox proportional hazard regression was used to determine potential significant prognostic factors of IBC. A nomogram was then constructed to evaluate patient survival based on certain variables. Univariate and multivariate analyses revealed that race $(p<0.001)$, M stage $(p<0.001)$, surgery $(p=0.010)$, chemotherapy (CT) $(p<0.001)$, tumor size $(p=0.010)$, estrogen receptor $(p<0.001)$, progesterone receptor $(p=0.04)$, and human epidermal growth factor receptor $2(p<0.001)$ were all independent risk factors. The concordance index (C-index) of the nomogram was 0.735 , which showed good predictive efficiency. Survival analysis indicated that IBC patients without CT had poorer survival than those with $\mathrm{CT}(p<0.001)$. Stratified analyses showed that modified radical mastectomy (MRM) had significant survival advantages over non-MRM in patients with stage IV IBC $(p=0.031)$. Patients treated with or without CT stratified by stage III and stage IV showed better survival than those without stage III and IV $(p<0.001)$. Trimodality therapy resulted in better survival than surgery combined with CT or $\mathrm{CT}$ alone $(p<0.001)$. Competing risk analysis also showed the same results. The nomogram was effectively applied to predict the 1,3 and 5-year survival of IBC. Our nomogram showed relatively good accuracy with a C-index of 0.735 and is a visualized individually predictive tool for prognosis. Treatment strategy greatly affected the survival of patients. Trimodality therapy was the preferable therapeutic strategy for IBC. Further prospective studies are needed to validate these findings. 
Introduction

39

40

41

42

Inflammatory breast carcinoma (IBC) is an uncommon subtype of locally advanced breast cancer with a poor prognosis and is characterized by aggressive behavior and rapid progression (Robertson et al., 2010). Management involves the coordination of multidisciplinary treatment and usually includes neoadjuvant chemotherapy, ablative surgery if a tumor-free resection margin is expected and locoregional radiotherapy. This multimodal therapeutic approach has significantly improved patient survival. However, the median overall survival (OS) among women with IBC is still poor. Clinically, IBC is characterized by the rapid onset of breast warmth, erythema, and edema often without a well-defined mass. Along with extensive breast involvement, women with IBC often have early involvement of the axillary lymph nodes. The median survival time of IBC treated by surgery alone or combined with radiotherapy (RT) is less than 15 months and the local recurrence rate is approximately $50 \%$ (Zucali et al., 1976). Despite the significant progress made in the treatment of this aggressive form of breast cancer, most women with IBC will relapse and succumb to this disease. Limited research with small cohorts have focused on the prognostic value of RT and CT (Semiglazov et al., 2007; Jardel et al., 2018). A few studies have analyzed the clinicopathological features related to OS, such as lymph node status and human epidermal growth factor receptor 2 (Her-2) status (Dawood et al., 2008; Wecsler et al., 2015). It is important to evaluate the effect of trimodality therapy in IBC patients. In addition, high risk patients who need more aggressive locoregional treatment are not well-defined in the literature. The current investigation aimed to determine the clinicopathological features of IBC and identify reliable and 
58

59

60

61 subtype is clinically useful for predicting survival outcome in IBC (Wu et al., 2019). The

62 identifying factor of survival was related to race/ethnic group (Il'Yasova et al., 2011) and the

63 overall survival IBC patients from the SEER database has been reported to be associated with

64 socioeconomic position (Liu et al., 1998; Schlichting et al., 2012). In contrast, we determined the

65 independent prognostic factors of IBC, and then constructed a nomogram.

66

67

68

69

70

71 72 procedures,
A nomogram, a statistic-based tool to calculate the risk of clinicopathological features of a cancer, has been widely developed to evaluate survival in cancer patients ( Zhou et al., 2015; Sun et al., 2017). It has been proved to be accurate with the advantage of visualization and quantification with a friendly interface for doctors and patients. In this study, a nomogram was constructed to predict 1-, 3-, and 5-year cancer-specific survival (CSS) according to multivariate analyses based on this large cohort, and to identify the exact therapeutic effect of various surgical and

CT. 
Materials and Methods

74

75

76

77

\section{Data source and inclusion criteria}

We retrieved patient data from the SEER database (SEER*Stat 8.3.5, http://seer.cancer.gov/). The SEER database is free to the public and is updated annually, with routinely collected general messages from patients, primary tumor characteristics, treatments, survival and follow-up, etc. In this study, the data were updated in November 2016, and released on April 16, 2018. The target population downloaded from the database was from 1973 to 2015 . We extracted IBC patients between 2010 and 2015 as data on Her-2 status was released from 2010. Patients were included in this study if they met the following criteria: patients who had IBC with the International Classification of Diseases for Oncology, $3^{\text {rd }}$ edition (ICD-O-3): 8530 as the histopathology code, positive histology, complete survival month flag and active follow-up. Patients confirmed by autopsy only or death certificate only and those with invalid follow-up data were excluded. In total, 883 patients were selected for further analysis in this study. Data downloaded from SEER did not require patients' informed consent and may be reproduced or copied without permission. This study was approved to be exempted by the Institutional Review Committee of Zhongshan Hospital (Fudan University, Shanghai, China).

\section{Endpoints and statistical analysis}

OS was calculated from the date of first diagnosis to the date of death or last contact. CSS was measured from the date of first diagnosis to the date of death due to breast carcinoma. However, 
92 patients who died of other causes were considered censoring events. All statistical analyses were

93 performed using SPSS 24.0 (IBM Corp, Armonk, NY, USA) or R package 3.4.4 software

94 (http://www.r-project.org/; R Foundation for Statistical Computing, Vienna, Austria). Cox

95

96

97

98

99

100

101

102

103

104

105 proportional hazard regression was applied to identify significant prognostic factors with hazard ratios (HRs) and 95\% confidence intervals (95\% CIs). Variables in the univariate analysis with $p$ values $<0.05$ were selected for multivariate analysis using backward stepwise regression (likelihood ratio). The nomogram was constructed based on multivariate analysis results, and was evaluated by the concordance index (C-index), the calibration curves and receiver operating characteristics (ROC) curves. The value of the C-index ranges from 0.5 to 1.0 and a larger value indicates better accuracy of predicting efficiency (Koepsell TD, 2014; Ma et al., 2018). The calibration curves were based on 1,000 times bootstrap resampling. The Kaplan-Meier method and log-rank test were performed to obtain survival curves and the differences between groups were compared using $\mathrm{R}$ project 3.4 .4 with the survival package. We also performed competing risk analysis. All differences with $p$ values $<0.05$ were considered to be statistically significant. 


\section{Patient characteristics}

108

109

110

111

112

113

114

115

116

117

118

119

120

121

122

123

124

125

A total of 883 patients diagnosed with IBC were extracted from the SEER database between 2010 and 2015 according to the abovementioned inclusion criteria. The results of univariate/multivariate analyses based on CSS and OS as well as 5-year OS are summarized in Tables 1-3, respectively. The median age of the IBC patients was 57 (range, 22-97) years. The majority $(693,78.5 \%)$ were White. The 5-year OS of White vs. Black patients was $49.4 \%$ vs. $29.7 \%$ and the HR for CSS was $1.90(95 \%$ CI $1.43-2.53, p<0.001)$. The 3 - and 5-year OS of patients with IBC was $54.4 \%$ and $47.4 \%$, respectively. The median OS was 43.0 (95\% CI, 32.4-53.6) months. Tumor staging followed the criteria of the $7^{\text {th }}$ edition of American Joint Committee on Cancer (AJCC) for breast cancer. Most patients $(572,64.8 \%)$ were stage III, while 311 (35.2\%) were stage IV. Patients who were treated with surgery accounted for $58.2 \%$, of which, 352 (39.9\%) patients underwent MRM and $162(18.3 \%)$ patients were treated by non-MRM including $30(3.0 \%)$ with breast-conserving surgery, $118(13.4 \%)$ with total (simple) mastectomy, $9(1.0 \%)$ with radical mastectomy and 5 $(0.9 \%)$ with extended radical mastectomy. The HR and $95 \% \mathrm{CI}$ of the non-MRM group vs. the MRM group was $0.41(0.30-0.57)$ vs. $0.33(0.26-0.43)$ for CSS. RT (396/883, 44.8\%) and CT (721/883, 81.7\%) were also main treatments for patients with IBC.

Univariate analysis of CSS for IBC using Cox regression analysis demonstrated that age ( $p$ $=0.016)$, race $(p<0.001), \mathrm{N}$ stage $(p<0.001), \mathrm{M}$ stage $(p<0.001)$, surgical modality $(p<$ $0.001)$, radiation status $(p<0.001)$, CT status $(p<0.001)$, tumor size $(p=0.015)$, estrogen 
126

127

128

129

130

131

132

receptor (ER) $(p<0.001)$, progesterone receptor (PR) $(p<0.001)$, Her-2 $(p<0.001)$ and marital status ( $p=0.002$ ) were all significant prognostic factors, and corresponded to those for OS. All the significantly different variables were included in the multivariate analyses of CSS and OS (Table 1 and Table 2). Finally, race (Black, HR = 1.77), M stage (M1, HR =2.93), surgery (nonMRM, HR = 0.74; MRM, HR =0.60), CT status $(Y e s, H R=0.46)$, tumor size $(2-5 \mathrm{~cm}, \mathrm{HR}=$ 0.91; $>5 \mathrm{~cm}, \mathrm{HR}=1.48$; Diffuse, HR = 1.39), ER (Positive, HR = 0.52), PR (Positive, HR = 0.64 ) and Her-2 (Positive, HR $=0.12$ ) were confirmed as independent prognostic factors of IBC.

(Figure 1A). The points from each independent prognostic factor listed in the nomogram were added together. The 1, 3 and 5-year CSS rates for patients with IBC were estimated by applying three point scales at the bottom of the nomogram, respectively. The C-index of this model was 0.735 which showed an excellent predictive efficiency. We performed a calibration plot to resample the validation of the nomogram based on 1000 bootstraps to confirm the agreement between the actual and predicted 1,3 and 5-year CSS rates (Figure 1B-D). A series of receiver operating characteristics (ROC) curves were performed to verify the accuracy of the nomogram (Figure S1A-C).

\section{Survival analyses in subgroups of patients with inflammatory breast carcinoma}

Survival curves were used to compare the CSS of patients with IBC according to the following parameters: TNM stage, CT, surgical modality, and treatment pattern by subgroup analyses. All 883 patients classified by different surgical modalities achieved statistically significant survival ( $p$ 
$146<0.001)$ (Figure 2A). There was no significant difference in CSS between the MRM and non-

147 MRM group $(p=0.23)$ (Figure 2B). When the patients were stratified by TNM stage III and IV, 148 the $p$ values were 0.5 (Figure 2C) and 0.031 (Figure 2D), respectively. Patients treated with CT 149 had better survival $(p<0.001)$ (Figure 3A). Competing risk analysis showed the same results 150 (Figure S2). Furthermore, subgroup analyses stratified by TNM stages (Figure 3B, 3C) indicated 151 that patients in the TNM stages benefitted from CT in terms of CSS $(p<0.001)$. When patients 152 were classified by different treatment patterns (Figure 4), such as surgery alone, RT alone, CT 153 alone, surgery combined with RT, surgery combined with CT, RT combined with CT, or surgery 154 combined with RT and CT, there were significant differences between them. The results indicated 155 that surgery combined with RT and CT was the optimal treatment pattern. A further subgroup 156 analysis of the treatment modalities was performed and the treatments which resulted in greatest 157 survival (Figure 4) were surgery combined with RT and CT, followed by CT and RT, and CT 158 alone with significant differences between the three groups $(p<0.001)$. Competing analysis 159 revealed the same results (Figure S3).

\section{Discussion}

IBC is uncommon and the most lethal form of breast carcinoma, and leads to a worse prognosis compared with other forms of breast carcinoma, with significantly lower OS, (3-year OS, 42\% vs. $85 \%$ ) (Chang et al., 1998). Treatment with multimodal therapies has significantly improved the survival of patients with IBC in recent years, especially when targeted therapy is available (Gianni 
166 et al., 1997). The 3- and 5-year OS of patients with IBC was 54.4\% and 47.4\% in this study, which

167 is consistent with the above previously published reports. To date, there are still many uncertainties

168 regarding the optimal treatment of IBC. As a result of small sample sizes, various treatment 169 patterns, and variable response criteria, evidence-based management has been determined largely by institutional experience or based on other types of breast carcinoma (Panades et al., 2005). This study analyzed the survival of a large cohort of patients with IBC based on the SEER database and confirmed eight independent prognostic factors including race, $\mathrm{M}$ stage, surgical modality, CT status, tumor size, ER, PR and Her-2 status, based on the nomogram which will provide the first comprehensive evaluation profile to help physicians make a reasonable treatment decision and estimate prognosis in IBC patients. important treatment strategy for patients with breast carcinoma who are either ER or PR positive. However, information on endocrine therapy in IBC patients is not available in the SEER database. Her-2, is an oncogene and is overexpressed and/or amplified in approximately $30 \%$ of patients with breast carcinoma (Slamon et al., 1987), and is related to increased aggressiveness, a higher recurrence rate and mortality (Romond EH, 2005). Furthermore, it was reported that Her-2 was overexpressed and amplified by $36-60 \%$ in patients with IBC than in those with non-IBC (Parton et al., 2004; Guerin et al., 1990). Patients with positive expression of Her-2 showed shorter recurrence-free survival and OS compared with patients without Her-2 amplification in a study of breast carcinoma by Slamon et al (Slamon et al., 1987). However, the role of Her-2 as a poor 
prognostic factor in breast carcinoma was altered by the introduction of trastuzumab, a monoclonal

187

190

191

192

193

194

195

196

197

198

199

200

201

202

203

204

205

antibody which targets the Her-2 receptor (Wecsler et al., 2015). Data from the SEER database showed that IBC patients with HoR+/Her2- subtype had poorer breast cancer-specific survival and OS than those with HoR+/Her-2 subtype (Wu et al., 2019). Her2-positive status in this study had a protective effect in patients with IBC, which was in accordance with previous reports based on the SEER database (Li et al., 2017).

Surgery and RT are important locoregional therapies in IBC. However, either surgery alone (Fields et al., 1989) or RT alone (Jaiyesimi et al., 1992) produced disappointing results in the treatment of IBC. Even though combining surgery with RT significantly improves locoregional control, with disease-free survival as high as 24 months (Perez \& Fields, 1987), the median survival of the two treatments combined ranges from 7 to 29 months and was not significantly different from either treatment alone. Data on IBC patients from the SEER database showed that surgery combined with RT significantly improved 5-year OS by 15\% (Muzaffar et al., 2018). However, RT was not an independent prognostic factor of CSS in IBC patients. Researchers have now realized that breast cancer is a systemic disease. Locoregional therapy is not enough to prevent distant metastasis. Therefore, systemic CT has been combined with surgery, RT, hormonal therapy and molecular targeted therapy. In this study, IBC patients treated with CT vs. without CT achieved a 5 -year OS of $52.1 \%$ vs. $25.1 \%$ and a median OS of 63.0 vs. 15.0 months, respectively. These results are consistent with previous reports which showed that CT improved the 5-year OS of patients with IBC to $30-40 \%$ (Fields et al., 1989). Interestingly, our stratified analysis of surgical 
206

207

208

209

210

211

212

213

214

215

216

217

218

219

220

221

222

modalities based on TNM stage revealed that stage IV patients achieved a significant difference in survival when treated with MRM compared with non-MRM. Nevertheless, no difference was observed in stage III IBC patients. In this study, 118 (72.8\%) patients in the non-MRM group underwent total (simple) mastectomy. Only $9(1.0 \%)$ patients underwent radical mastectomy and $5(0.9 \%)$ patients underwent extended radical mastectomy. MRM is the most commonly used surgical approach of choice, as it allows both removal of the main tumor mass and adjacent glandular tissue with suspected infiltration and multifocality, and a sentinel axillary lymph node. This procedure significantly reduces primary tumor burden and peripheral subclinical lesions. Theoretically, it should bring marked benefits to both stage III and IV patients. A comprehensive explanation to rationalize these observations is still required. Lymph node status is also an important prognostic factor in IBC (Liang et al., 2015) and positive node status was reported to be a negative prognostic factor (Wecsler et al., 2015). However, $\mathrm{N}$ stage in our study was not statistically significant in multivariate analysis. A total of 355 patients in our study received no surgery; the $\mathrm{N}$ stage in these patients was categorized only by clinical features without pathology confirmation. We assume that inaccurate $\mathrm{N}$ stage or less important than $\mathrm{M}$ stage in multivariate Cox regression analysis could be the reasons why lymph node status was not included as a prognostic factor of IBC.

Trimodality treatment is recommended following the sequence of CT (including trastuzumab and hormonal therapy when necessary), then surgery and RT (Robertson et al., 2010). Two prospective randomized trials involving 68 patients with $\mathrm{IBC}$ treated with three cycles of CAF 
226 (cyclophosphamide, doxorubicin, and 5-fluorouracil) or CEF (cyclophosphamide, epirubicin, and

227 5-fluorouracil) followed by surgery, adjuvant therapy, and RT, reported a 5-year OS of 44\% and

228 a 10-year OS of 32\% (Baldini et al., 2004). Our subgroup analysis based on treatment group

229 including CT alone, CT combined with surgery, and CT combined with surgery and RT showed

230 the advantage of trimodality treatment on survival. It was reported that patients with ER-negative

231 IBC benefitted from paclitaxel together with anthracycline-based regimens and obtained improved

232 progression-free survival and OS (Cristofanilli et al., 2007; Cristofanilli et al., 2004). The optimal

233 chemotherapeutic regimens and optimal combination of targeted treatments deserve further

234 investigation.

To our knowledge, no nomogram model has been established to predict the survival of patients with IBC. As $34.4 \%$ of deaths are not attributed to primary breast cancer, CSS was used

237 here to exclude the potential impact of other factors. It is worth mentioning that our study included 238 a large sample size of 883 patients which is difficult to achieve in a single institute clinical trial. In addition to race, Her-2 and hormone receptor status have been reported as prognostic factors for OS in IBC. All prognostic factors in the present study have not been reported in association with CSS. This nomogram included surgical modalities (MRM vs. non-MRM), and MRM was 242 confirmed to be a protective factor for CSS compared to non-MRM. The nomogram with 243 predictive accuracy was constructed based on comprehensive clinical pathological features in this 244 study. However, there are some limitations that should be noted. The SEER database did not 245 provide sufficient information on systemic therapy, such as CT regimens in patients with IBC, 
246 Her2-positive patients treated with or without trastuzumab, and hormone receptor-positive patients

247 treated with or without hormonal therapy. Furthermore, limited information on local therapy, such

248 as the sequence of RT and surgery, radiation dose and radiation fields, constrained our

249 identification of more prognostic factors associated with detailed treatment information.

250 Additionally, a prospective study should be conducted to validate the reliability of these results.

\section{Conclusions}

252 A nomogram can be effectively applied to predict the 1-, 3- and 5-year survival of IBC patients.

253 Our nomogram showed relatively good accuracy with a C-index of 0.735 and is a visualized

254 individually predictive tool for prognosis. Treatment strategy greatly affected the survival of

255 patients. Trimodality therapy was the preferable therapeutic strategy for IBC. Further prospective

256 studies are needed to validate these findings.

\section{Acknowledgements}

258 We thank Dr. Huang Yiwei for his partial statistical analysis in this study. This work was

259 supported by Luoyang City Science and Technology Plan Medical and Health Project (Grant No.

260 1721001A-2) and the Youth Program of National Natural Science Foundation of China (Grant

261 No. 81502005).

262 Conflicts of interest

263 The authors declare no competing interests. 


\section{References:}

266

267

268

269

270

271

272

273

274

275

276

277

282

283

Baldini, E., Gardin, G., Evagelista, G., Prochilo, T., Collecchi, P., and Lionetto, R. 2004. Long-term results of combined-modality therapy for inflammatory breast carcinoma. Clinical Breast Cancer 5:358-363.

Chang, S., Parker, S.L., Pham, T., Buzdar, A.U., and Hursting, S.D. 1998. Inflammatory breast carcinoma incidence and survival: the surveillance, epidemiology, and end results program of the National Cancer Institute, 19751992. CANCER 82:2366-2372.

Cristofanilli, M., Gonzalez-Angulo, A.M., Buzdar, A.U., Kau, S.W., Frye, D.K., and Hortobagyi, G.N. 2004. Paclitaxel improves the prognosis in estrogen receptor negative inflammatory breast cancer: the M. D. Anderson Cancer Center experience. Clinical Breast Cancer 4:415-419.

Cristofanilli, M., Valero, V., Buzdar, A.U., Kau, S.W., Broglio, K.R., Gonzalez-Angulo, A.M., Sneige, N., Islam, R., Ueno, N.T., Buchholz, T.A., Singletary, S.E., and Hortobagyi, G.N. 2007. Inflammatory breast cancer (IBC) and patterns of recurrence: understanding the biology of a unique disease. CANCER 110:1436-1444. $10.1002 /$ cncr. 22927.

Dawood, S., Broglio, K., Gong, Y., Yang, W.T., Cristofanilli, M., Kau, S.W., Meric-Bernstam, F., Buchholz, T.A., Hortobagyi, G.N., and Gonzalez-Angulo, A.M. 2008. Prognostic significance of HER-2 status in women with inflammatory breast cancer. CANCER 112:1905-1911. 10.1002/cncr.23350.

Gianni, L., Eiermann, W., Semiglazov, V., Manikhas, A., Lluch, A., Tjulandin, S., Zambetti, M., Vazquez, F., Byakhow, M., Lichinitser, M., Climent, M.A., Ciruelos, E., Ojeda, B., Mansutti, M., Bozhok, A., Baronio, R., Feyereislova, A., Barton, C., Valagussa, P., and Baselga, J. 2010. Neoadjuvant chemotherapy with trastuzumab followed by adjuvant trastuzumab versus neoadjuvant chemotherapy alone, in patients with HER2-positive locally advanced breast cancer (the NOAH trial): a randomised controlled superiority trial with a parallel HER2-negative cohort. LANCET 375:377-384. 10.1016/S0140-6736(09)61964-4.

Peer) reviewing PDF | (2019:04:36963:1:1:NEW 30 Jul 2019) 
293

295

296

Guerin, M., Sheng, Z.M., Andrieu, N., and Riou, G. 1990. Strong association between c-myb and oestrogen-receptor expression in human breast cancer. ONCOGENE 5:131-135.

Hance, K.W., Anderson, W.F., Devesa, S.S., Young, H.A., and Levine, P.H. 2005. Trends in Inflammatory Breast Carcinoma Incidence and Survival: The Surveillance, Epidemiology, and End Results Program at the National Cancer Institute. JNCI: Journal of the National Cancer Institute 97:966-975. 10.1093/jnci/dji172.

Il'Yasova, D., Siamakpour-Reihani, S., Akushevich, I., Akushevich, L., Spector, N., and Schildkraut, J. 2011. What can we learn from the age- and race/ethnicity- specific rates of inflammatory breast carcinoma? Breast Cancer Res Treat 130:691-697. 10.1007/s10549-011-1719-4.

Jaiyesimi, I.A., Buzdar, A.U., and Hortobagyi, G. 1992. Inflammatory breast cancer: a review. JOURNAL OF CLINICAL ONCOLOGY 10:1014-1024. 10.1200/JCO.1992.10.6.1014.

Jardel, P., Alami, Z., Vignot, S., Creisson, A., Danhier, S., Geffrelot, J., Levy, C., Kammerer, E., Lebrun, J.F., and Thariat, J. 2018. Radiothérapie des cancers du sein inflammatoires. BULLETIN DU CANCER 105:415-425. 10.1016/j.bulcan.2017.12.008.

Koepsell TD, W.N. 2014. Epidemiologic Methods: Studying the Occurrence of Illness. UK: Oxford University Press.

Li, J., Xia, Y., Wu, Q., Zhu, S., Chen, C., Yang, W., Wei, W., and Sun, S. 2017. Outcomes of patients with inflammatory breast cancer by hormone receptor- and HER2-defined molecular subtypes: A population-based study from the SEER program. Oncotarget 8:49370-49379. 10.18632/oncotarget. 17217.

Liu, L., Deapen, D., and Bernstein, L. 1998. Socioeconomic status and cancers of the female breast and reproductive organs: a comparison across racial/ethnic populations in Los Angeles County, California (United States). Cancer Causes Control 9:369-380. 
321

322

323

324

325

326

327

328

329

330

331

332

333

334

335

336

337

338

339

340

341

342

343

344

345

346

347

Ma, K., Sun, F., Yang, X., Wang, S., Wang, L., Jin, Y., Shi, Y., Jiang, W., Zhan, C., and Wang, Q. 2018. Prognosis of patients with primary malignant main stem bronchial tumors: 7,418 cases based on the SEER database. Onco Targets Ther 11:83-95. 10.2147/OTT.S142847.

Muzaffar, M., Johnson, H.M., Vohra, N.A., Liles, D., and Wong, J.H. 2018. The Impact of Locoregional Therapy in Nonmetastatic Inflammatory Breast Cancer: A Population-Based Study. Int J Breast Cancer 2018:6438635. $10.1155 / 2018 / 6438635$.

Panades, M., Olivotto, I.A., Speers, C.H., Shenkier, T., Olivotto, T.A., Weir, L., Allan, S.J., and Truong, P.T. 2005. Evolving Treatment Strategies for Inflammatory Breast Cancer: A Population-Based Survival Analysis. 23:1941-1950. 10.1200/JCO.2005.06.233.

Parton, M., Dowsett, M., Ashley, S., Hills, M., Lowe, F., and Smith, I.E. 2004. High incidence of HER-2 positivity in inflammatory breast cancer. BREAST 13:97-103. 10.1016/j.breast.2003.08.004.

Perez, C.A., and Fields, J.N. 1987. Role of radiation therapy for locally advanced and inflammatory carcinoma of the breast. Oncology (Williston Park) 1:81-94.

Robertson, F.M., Bondy, M., Yang, W., Yamauchi, H., Wiggins, S., Kamrudin, S., Krishnamurthy, S., Le-Petross, H., Bidaut, L., Player, A.N., Barsky, S.H., Woodward, W.A., Buchholz, T., Lucci, A., Ueno, N., and Cristofanilli, M. 2010. Inflammatory Breast Cancer: The Disease, the Biology, the Treatment. CA: A Cancer Journal for Clinicians 60:351-375. 10.3322/caac.20082.

Schlichting, J.A., Soliman, A.S., Schairer, C., Banerjee, M., Rozek, L.S., Schottenfeld, D., Harford, J.B., and Merajver, S.D. 2012. Association of inflammatory and noninflammatory breast cancer with socioeconomic characteristics in the Surveillance, Epidemiology, and End Results database, 2000-2007. Cancer Epidemiol Biomarkers Prev 21:155-165. 10.1158/1055-9965.EPI-11-0833.

Peer) reviewing PDF | (2019:04:36963:1:1:NEW 30 Jul 2019) 
349

350

351

352

353

354

355

356

357

358

359

360

361

362

363

364

365

366

367

368

369

370

371

372

373

374
Semiglazov, V.F., Zernov, K., Bozhok, A.A., Petrovskii, S.G., Ivanova, O.A., Orlov, A.A., Barash, N., Paltuev, R.M., Donskikh, R.V., Damenia, A.O., Kletsel', A.E., Kochetova, I.A., and Efimenko, A.V. 2007. [Inflammatory breast carcinoma and prognosticators of efficacy of primary chemotherapy]. Vopr Onkol 53:21-25.

Slamon, D.J., Clark, G.M., Wong, S.G., Levin, W.J., Ullrich, A., and McGuire, W.L. 1987. Human breast cancer: correlation of relapse and survival with amplification of the HER-2/neu oncogene. SCIENCE 235:177-182.

Ueno, N.T., Buzdar, A.U., Singletary, S.E., Ames, F.C., McNeese, M.D., Holmes, F.A., Theriault, R.L., Strom, E.A., Wasaff, B.J., Asmar, L., Frye, D., and Hortobagyi, G.N. 1997. Combined-modality treatment of inflammatory breast carcinoma: twenty years of experience at M. D. Anderson Cancer Center: Cancer Chemother Pharmacol. 1997;40(4):321-9.

Wecsler, J.S., Tereffe, W., Pedersen, R.C., Sieffert, M.R., Mack, W.J., Cui, H., Russell, C.A., Woods, R.R., Viscusi, R.K., Sener, S.F., and Lang, J.E. 2015. Lymph node status in inflammatory breast cancer. 151:113-120. $10.1007 /$ s10549-015-3367-6.

Wu, S.G., Zhang, W.W., Wang, J., Dong, Y., Sun, J.Y., Chen, Y.X., and He, Z.Y. 2019a. Inflammatory breast cancer outcomes by breast cancer subtype: a population-based study. Future Oncology 15:507-516. 10.2217/fon2018-0677.

Wu, S.G., Zhang, W.W., Wang, J., Dong, Y., Sun, J.Y., Chen, Y.X., and He, Z.Y. 2019b. Inflammatory breast cancer outcomes by breast cancer subtype: a population-based study. Future Oncology 15:507-516. 10.2217/fon2018-0677.

Zucali, R., Uslenghi, C., Kenda, R., and Bonadonna, G. 1976. Natural history and survival of inoperable breast cancer treated with radiotherapy and radiotherapy followed by radical mastectomy. CANCER 37:1422-1431.

PeerJ reviewing PDF | (2019:04:36963:1:1:NEW 30 Jul 2019) 
Figure 1

Nomogram and calibration curves of patients with inflammatory breast carcinoma

(A) A nomogram for the prediction of 1-, 3- and 5-year cancer-specific survival in IBC patients. Calibration curves of the nomogram prediction of (B) 1-year, (C) 3-year, and (D) 5year survival of patients with IBC.

$\mathbf{A}$

\section{Points}

Race

ER

PR

HER2

Tumor size

M stage

Surgery

Chemotherapy

Total points

1-year survival

3-year survival

5-year survival
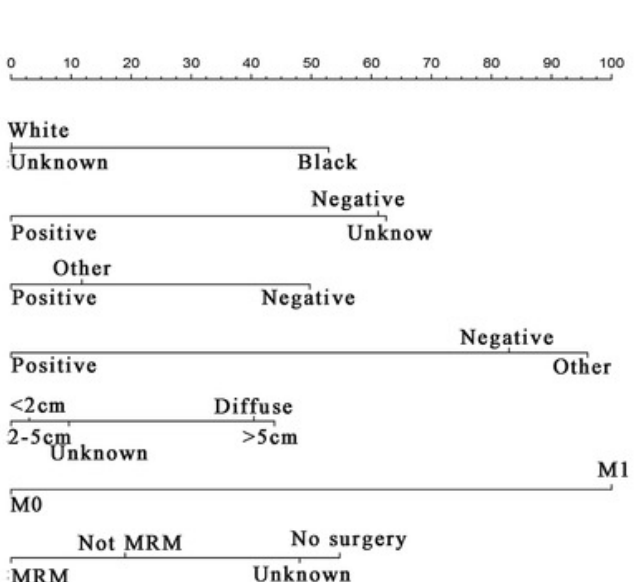

Yes
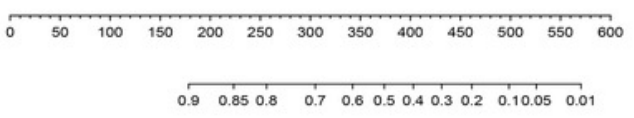

$\begin{array}{lllllllllllll}0.9 & 0.85 & 0.8 & 0.7 & 0.8 & 0.5 & 0.4 & 0.3 & 0.2 & 0.10 .05 & 0.01\end{array}$

$\begin{array}{lllllllllllllllll}0.9 & 0.85 & 0.8 & 0.7 & 0.6 & 0.5 & 0.4 & 0.3 & 0.2 & 0.10 .05 & 0.01\end{array}$
B
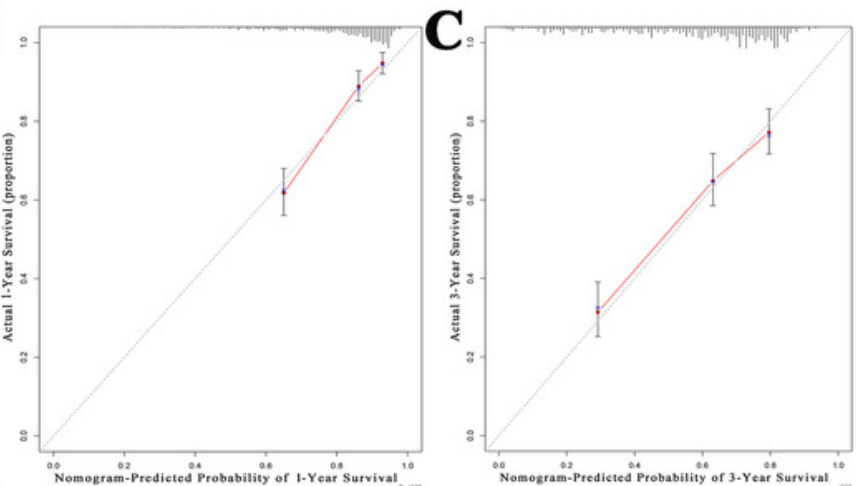

D

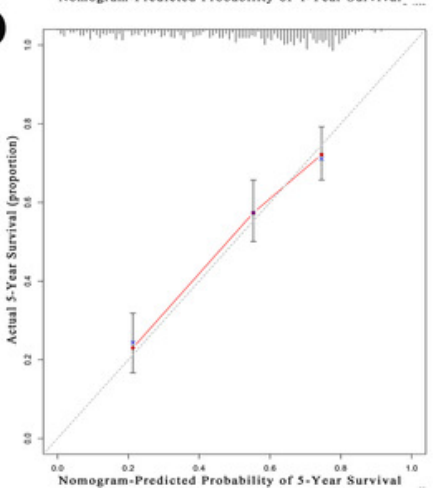


Figure 2

Survival analyses based on surgery and stratified analyses TNM stage

(A) Survival analyses of patients based on surgical modalities. (B) Survival analyses of patients with modified radical mastectomy (MRM) or non-MRM. Survival analyses of patients with MRM or non-MRM stratified by TNM stage. Five-year survival (C) stage III (MRM vs. nonMRM, $68 \%$ vs. $64.3 \%$ ). (D) stage IV (MRM vs. non-MRM, $43.2 \%$ vs. $18.9 \%$ ). 

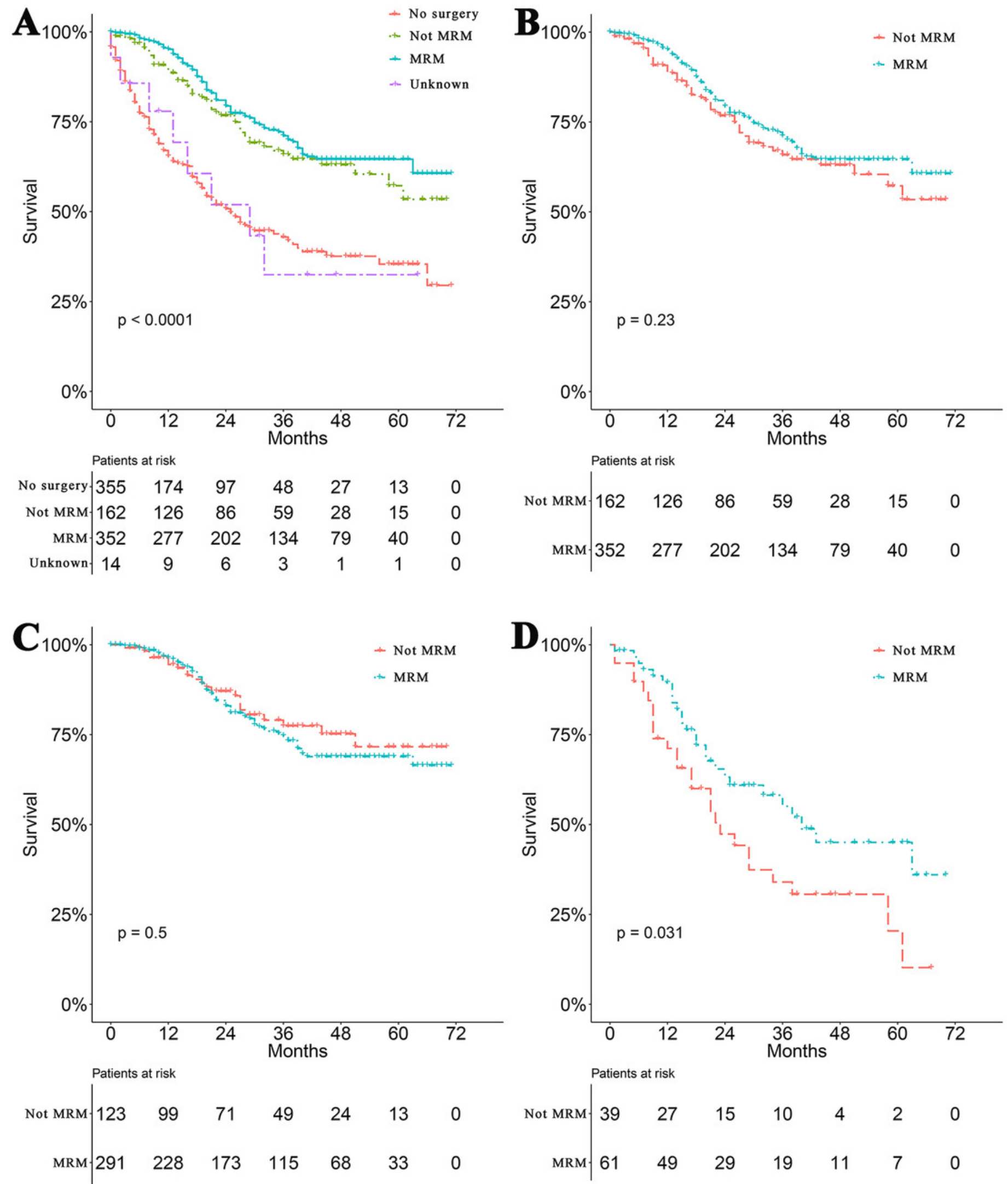
Figure 3

Survival analyses based on chemotherapy and stratified analyses based on TNM stage

(A) Survival analyses of patients with IBC treated with or without chemotherapy (CT). Survival analyses of patients with or without CT stratified by TNM stage. (B) stage III (Yes vs. No, $70.7 \%$ vs. $37.4 \%$ ) (C) stage IV (Yes vs. No, $39.7 \%$ vs. $8.3 \%$ ).
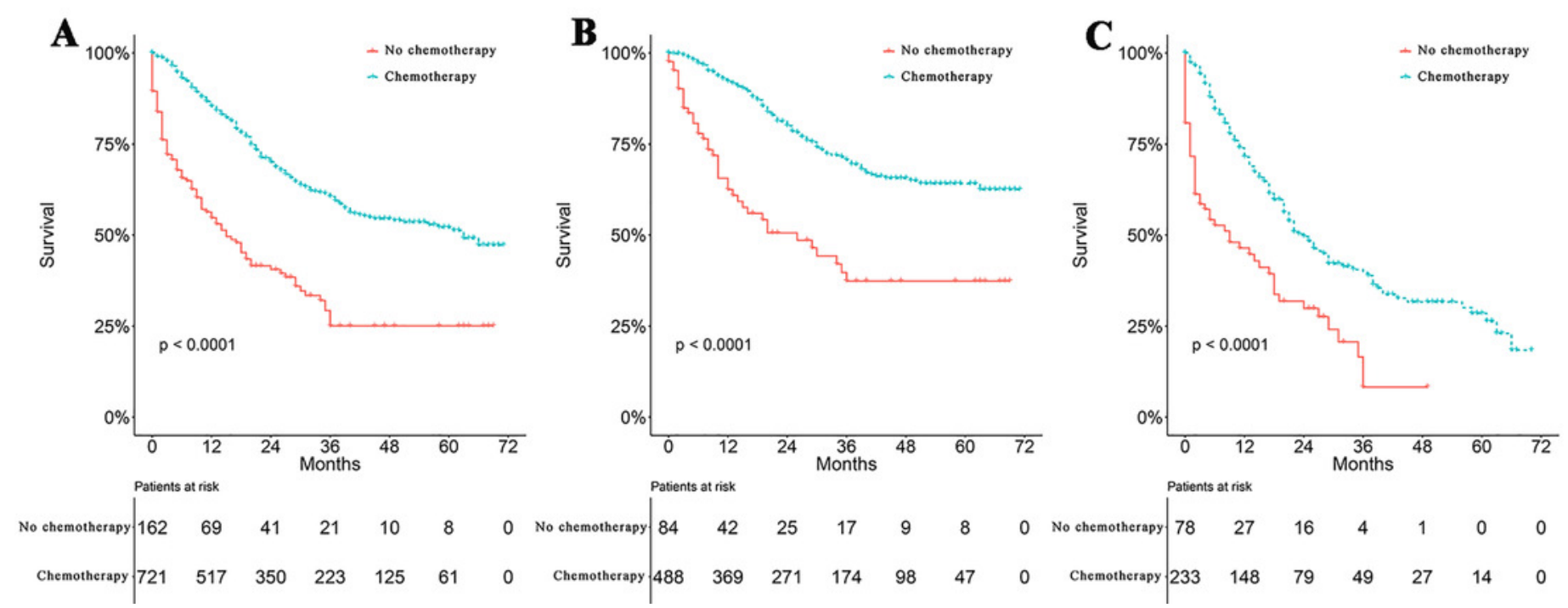
Figure 4

Survival analyses of patients with IBC treated with different strategies 


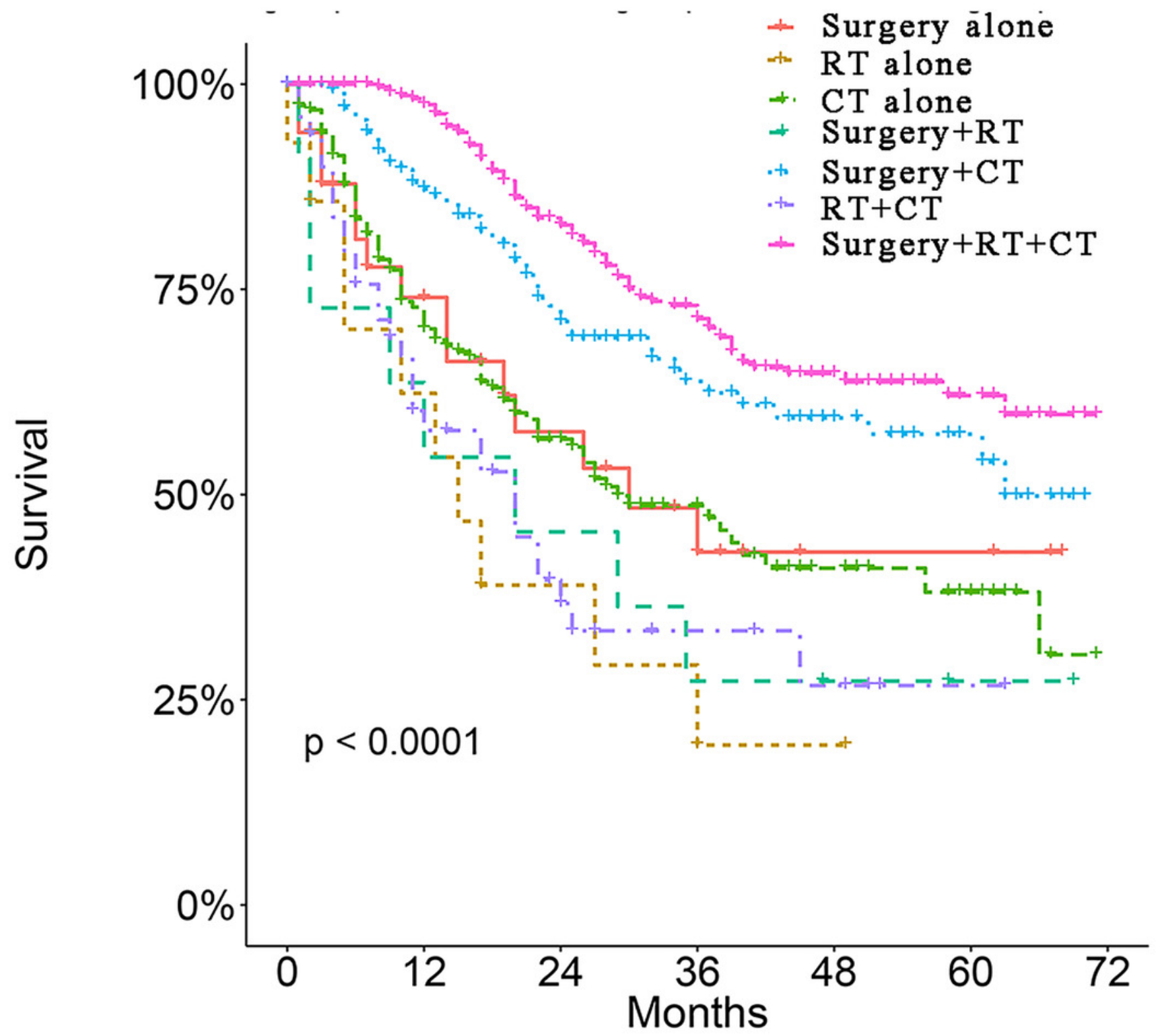

Patients at risk

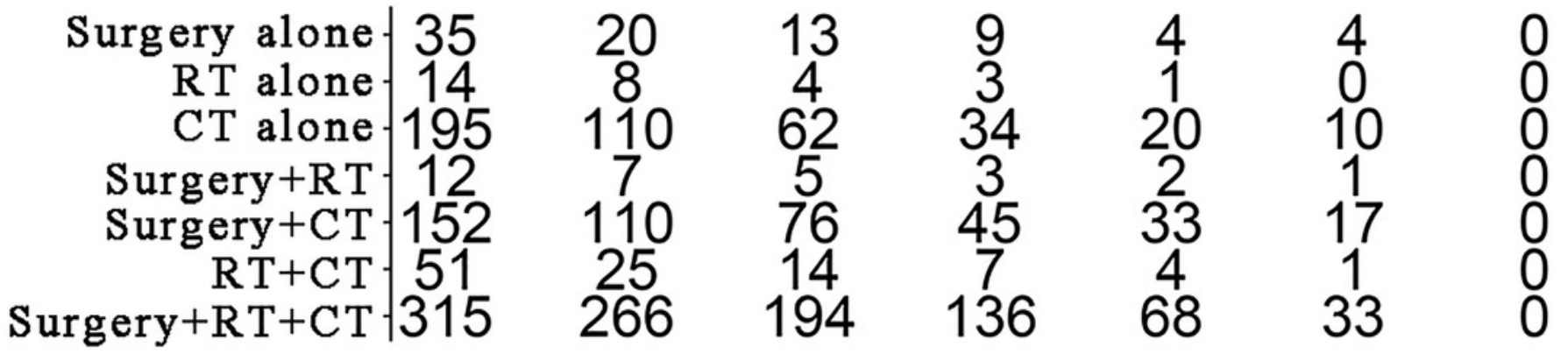




\section{Table $\mathbf{1}$ (on next page)}

Patient characteristics and analyses based on cancer specific survival status 
1 Table 1. Patient characteristics and analyses based on cancer specific survival status

\begin{tabular}{|c|c|c|c|c|c|}
\hline \multirow[t]{2}{*}{ Patient characteristics } & \multirow{2}{*}{$\begin{array}{c}\text { Number } \\
(\%)\end{array}$} & \multicolumn{2}{|c|}{ Univariate analysis } & \multicolumn{2}{|c|}{ Multivariate analysis } \\
\hline & & HR $(95 \%$ CI $)$ & P-value & $\mathrm{HR}(95 \% \mathrm{CI})$ & P-value \\
\hline Age & & & 0.016 & & \\
\hline$<50$ & $234(26.5)$ & $1[$ Reference $]$ & & & \\
\hline $50-59$ & $262(29.7)$ & $1.03(0.76-1.40)$ & 0.857 & & \\
\hline $60-69$ & $199(22.5)$ & $1.10(0.79-1.52)$ & 0.583 & & \\
\hline$\geq 70$ & $188(21.3)$ & $1.59(1.15-2.18)$ & 0.005 & & \\
\hline Race & & & $<0.001$ & & $<0.001$ \\
\hline White & $693(78.5)$ & $1[$ Reference $]$ & & $1[$ Reference $]$ & \\
\hline Black & $123(13.9)$ & $1.90(1.43-2.53)$ & $<0.001$ & $1.77(1.31-2.38)$ & $<0.001$ \\
\hline Unknown & $67(7.6)$ & $1.10(0.71-1.68)$ & 0.680 & $1.09(0.70-1.68)$ & 0.705 \\
\hline $\mathrm{N}$ stage & & & $<0.001$ & & \\
\hline N0 & $144(16.3)$ & $1[$ Reference $]$ & & & \\
\hline N1 & $372(42.1)$ & $1.49(1.02-2.18)$ & 0.038 & & \\
\hline $\mathrm{N} 2$ & $138(15.6)$ & $1.38(0.89-2.16)$ & 0.152 & & \\
\hline N3 & $177(20.0)$ & $1.85(1.22-2.78)$ & 0.003 & & \\
\hline $\mathrm{N}_{\mathrm{X}}$ & $52(5.9)$ & $3.08(1.87-5.07)$ & $<0.001$ & & \\
\hline M stage & & & $<0.001$ & & $<0.001$ \\
\hline M0 & $572(64.8)$ & $1[$ Reference $]$ & & $1[$ Reference $]$ & \\
\hline M1 & $311(35.2)$ & $3.23(2.57-4.06)$ & $<0.001$ & $2.93(2.27-3.79)$ & $<0.001$ \\
\hline Surgery & & & $<0.001$ & & 0.010 \\
\hline
\end{tabular}




\begin{tabular}{|c|c|c|c|c|c|}
\hline No surgery & $355(40.2)$ & $1[$ Reference $]$ & & 1 [Reference] & \\
\hline Not MRM & $162(18.3)$ & $0.41(0.30-0.57)$ & $<0.001$ & $0.74(0.52-1.04)$ & 0.086 \\
\hline MRM & $352(39.9)$ & $0.33(0.26-0.43)$ & $<0.001$ & $0.60(0.45-0.82)$ & $<0.001$ \\
\hline Unknown & $14(1.6)$ & $0.96(0.47-1.96)$ & 0.911 & $1.11(0.54-2.30)$ & 0.778 \\
\hline Radiation & & & $<0.001$ & & \\
\hline No & $487(55.2)$ & $1[$ Reference $]$ & & & \\
\hline Yes & $396(44.8)$ & $0.57(0.45-0.72)$ & $<0.001$ & & \\
\hline Chemotherapy & & & $<0.001$ & & $<0.001$ \\
\hline No & $162(18.3)$ & $1[$ Reference] & & $1[$ Reference $]$ & \\
\hline Yes & $721(81.7)$ & $0.41(0.32-0.54)$ & $<0.001$ & $0.46(0.34-0.63)$ & $<0.001$ \\
\hline Tumor size & & & 0.015 & & 0.010 \\
\hline$<2 \mathrm{~cm}$ & $68(7.7)$ & 1 [Reference] & & $1[$ Reference $]$ & \\
\hline $2-5 \mathrm{~cm}$ & $186(21.1)$ & $0.90(0.55-1.47)$ & 0.664 & $0.91(0.55-1.50)$ & 0.715 \\
\hline$>5 \mathrm{~cm}$ & $257(29.1)$ & $1.40(0.89-2.20)$ & 0.151 & $1.48(0.94-2.35)$ & 0.093 \\
\hline Diffuse & $179(20.3)$ & $1.61(1.00-2.57)$ & 0.048 & $1.39(0.86-2.25)$ & 0.178 \\
\hline Unknown & 193(21.9) & $1.21(0.75-1.96)$ & 0.429 & $0.95(0.57-1.57)$ & 0.834 \\
\hline ER & & & $<0.001$ & & $<0.001$ \\
\hline Negative & $386(43.7)$ & 1 [Reference] & & 1 [Reference] & \\
\hline Positive & $455(51.5)$ & $0.54(0.42-0.68)$ & $<0.001$ & $0.52(0.37-0.71)$ & $<0.001$ \\
\hline Unknown & $42(4.8)$ & $1.30(0.82-2.07)$ & 0.267 & $1.22(0.37-4.06)$ & 0.746 \\
\hline PR & & & $<0.001$ & & 0.04 \\
\hline Negative & $499(56.5)$ & 1 [Reference] & & $1[$ Reference $]$ & \\
\hline Positive & $331(37.5)$ & $0.60(0.46-0.77)$ & $<0.001$ & $0.64(0.45-0.91)$ & 0.013 \\
\hline
\end{tabular}




$\begin{array}{lccccc}\text { Other* } & 53(6.0) & 1.28(0.84-1.95) & 0.261 & 0.61(0.22-1.69) & 0.337 \\ & & & <0.001 & & \\ \text { Negative } & 496(56.2) & 1 \text { [Reference] } & & 1[\text { Reference] } & \\ \text { Positive } & 311(35.2) & 0.49(0.38-0.65) & <0.001 & 0.12(0.04-0.39) & <0.001 \\ \text { Other* } & 76(8.6) & 1.26(0.87-1.83) & 0.221 & 1.16(0.66-2.03) & 0.617\end{array}$

Marital status

Married

Divorce or widow

Single

Unknown
400(45.3)

238(27)

201(22.8)

$44(5.0)$
0.002

1 [Reference]

$1.29(0.97-1.71) \quad 0.078$

$1.47(1.10-1.95) \quad 0.008$

$2.10(1.35-3.29) \quad<0.001$
2

Abbreviations:

$3 \mathrm{ER}=$ estrogen receptor; $\mathrm{PR}=$ progesterone receptor; HER2 = human epidermal growth factor receptor 2; MRM =

4 Modified radical mastectomy; ${ }^{*}$ Other $=$ borderline + unknown; Reference $=$ This group is set as a reference group.

6

7

8

9

10

11

12

13

14

15 


\section{Table 2 (on next page)}

Patient characteristics and analyses based on overall survival status 
1 Table 2. Patient characteristics and analyses based on overall survival status

\begin{tabular}{|c|c|c|c|c|c|}
\hline \multirow[t]{2}{*}{ Patient characteristics } & \multirow{2}{*}{$\begin{array}{c}\text { Number } \\
(\%)\end{array}$} & \multicolumn{2}{|c|}{ Univariate analysis } & \multicolumn{2}{|c|}{ Multivariate analysis } \\
\hline & & HR $(95 \%$ CI $)$ & P-value & HR $(95 \%$ CI $)$ & P-value \\
\hline Age & & & $<0.001$ & & \\
\hline$<50$ & $234(26.5)$ & $1[$ Reference $]$ & & & \\
\hline $50-59$ & $262(29.7)$ & $1.06(0.78-1.43)$ & 0.727 & & \\
\hline $60-69$ & $199(22.5)$ & $1.21(0.88-1.65)$ & 0.237 & & \\
\hline$\geq 70$ & $188(21.3)$ & $1.91(1.42-2.58)$ & $<0.001$ & & \\
\hline Race & & & $<0.001$ & & 0.003 \\
\hline White & $693(78.5)$ & $1[$ Reference $]$ & & 1 [Reference] & \\
\hline Black & $123(13.9)$ & $1.70(1.29-2.24)$ & $<0.001$ & $1.64(1.23-2.18)$ & 0.001 \\
\hline Unknown & $67(7.6)$ & $0.98(0.65-1.49)$ & 0.093 & $0.99(0.65-1.52)$ & 0.993 \\
\hline $\mathrm{N}$ stage & & & $<0.001$ & & \\
\hline No & $144(16.3)$ & $1[$ Reference $]$ & & & \\
\hline N1 & $372(42.1)$ & $1.35(0.95-1.91)$ & 0.096 & & \\
\hline $\mathrm{N} 2$ & $138(15.6)$ & $1.34(0.90-2.02)$ & 0.153 & & \\
\hline N3 & $177(20.0)$ & $1.64(1.12-2.40)$ & 0.011 & & \\
\hline $\mathrm{N}_{\mathrm{X}}$ & $52(5.9)$ & $3.21(2.04-5.05)$ & $<0.001$ & & \\
\hline M stage & & & $<0.001$ & & $<0.001$ \\
\hline M0 & $572(64.8)$ & $1[$ Reference $]$ & & $1[$ Reference $]$ & \\
\hline M1 & $311(35.2)$ & $2.98(2.40-3.69)$ & $<0.001$ & $2.58(2.03-3.29)$ & $<0.001$ \\
\hline Surgery & & & $<0.001$ & & 0.004 \\
\hline
\end{tabular}




\begin{tabular}{|c|c|c|c|c|c|}
\hline No surgery & $355(40.2)$ & 1 [Reference] & & 1 [Reference] & \\
\hline Not MRM & $162(18.3)$ & $0.39(0.29-0.54)$ & $<0.001$ & $0.71(0.51-0.99)$ & 0.043 \\
\hline MRM & $352(39.9)$ & $0.33(0.26-0.42)$ & $<0.001$ & $0.59(0.45-0.79)$ & $<0.001$ \\
\hline Unknown & $14(1.6)$ & $0.84(0.41-1.71)$ & 0.633 & $0.94(0.45-1.94)$ & 0.864 \\
\hline Radiation & & & $<0.001$ & & \\
\hline No & $487(55.2)$ & $1[$ Reference] & & & \\
\hline Yes & $396(44.8)$ & $0.57(0.46-0.71)$ & $<0.001$ & & \\
\hline Chemotherapy & & & $<0.001$ & & $<0.001$ \\
\hline No & $162(18.3)$ & $1[$ Reference] & & 1 [Reference] & \\
\hline Yes & $721(81.7)$ & $0.35(0.28-0.44)$ & $<0.001$ & $0.39(0.29-0.51)$ & $<0.001$ \\
\hline Tumor size & & & 0.025 & & 0.033 \\
\hline$<2 \mathrm{~cm}$ & $68(7.7)$ & $1[$ Reference] & & 1 [Reference] & \\
\hline $2-5 \mathrm{~cm}$ & $186(21.1)$ & $0.97(0.61-1.55)$ & 0.904 & $0.97(0.61-1.56)$ & 0.907 \\
\hline$>5 \mathrm{~cm}$ & $257(29.1)$ & $1.39(0.90-2.15)$ & 0.140 & $1.47(0.95-2.29)$ & 0.870 \\
\hline Diffuse & $179(20.3)$ & $1.63(1.04-2.55)$ & 0.035 & $1.43(0.90-2.56)$ & 0.131 \\
\hline Unknown & $193(21.9)$ & $1.33(0.85-2.09)$ & 0.218 & $1.07(0.66-1.72)$ & 0.786 \\
\hline ER & & & $<0.001$ & & \\
\hline Negative & $386(43.7)$ & $1[$ Reference $]$ & & 1 [Reference] & \\
\hline Positive & $455(51.5)$ & $0.59(0.47-0.73)$ & $<0.001$ & $0.56(0.41-0.76)$ & $<0.001$ \\
\hline Unknown & $42(4.8)$ & $1.33(0.85-2.07)$ & 0.211 & $1.02(0.34-3.05)$ & 0.972 \\
\hline PR & & & $<0.001$ & & 0.021 \\
\hline Negative & $499(56.5)$ & 1 [Reference] & & 1 [Reference] & \\
\hline Positive & $331(37.5)$ & $0.64(0.51-0.82)$ & $<0.001$ & $0.63(0.45-0.87)$ & 0.006 \\
\hline
\end{tabular}




\begin{tabular}{|c|c|c|c|c|c|}
\hline Other* & $53(6.0)$ & $1.31(0.88-1.95)$ & 0.19 & $0.70(0.28-1.75)$ & 0.445 \\
\hline Ter-2 & & & $<0.001$ & & $<0.001$ \\
\hline Negative & $496(56.2)$ & 1 [Reference] & & 1 [Reference] & \\
\hline Positive & $311(35.2)$ & $0.51(0.39-0.65)$ & $<0.001$ & $0.46(0.35-0.60)$ & $<0.001$ \\
\hline Other* & $76(8.6)$ & $1.27(0.89-1.81)$ & 0.18 & $1.12(0.66-1.88)$ & 0.677 \\
\hline Iarital status & & & 0.002 & & \\
\hline Married & $400(45.3)$ & $1[$ Reference $]$ & & & \\
\hline Divorce or widow & $238(27)$ & $1.47(1.14-1.90)$ & 0.003 & & \\
\hline Single & $201(22.8)$ & $1.40(1.06-1.84)$ & 0.017 & & \\
\hline Unknown & $44(5.0)$ & $1.91(1.22-2.97)$ & 0.004 & & \\
\hline
\end{tabular}

2 Other*= borderline + unknown.

3

4

5

6

7

8

9

10

11

12

13

14 
Table 3 (on next page)

Five-year overall survival of patients with IBC 
1 Table 3. List of 5-year overall survival for patients with inflammatory breast carcinoma

\begin{tabular}{|c|c|c|c|}
\hline \multirow[t]{2}{*}{ Patient characteristics } & \multirow{2}{*}{$\begin{array}{c}\text { Number } \\
(\%)\end{array}$} & \multicolumn{2}{|r|}{ Kaplan-Meier } \\
\hline & & $5-\mathrm{OS}(\%)$ & Median OS (months) \\
\hline \multicolumn{4}{|l|}{ Age } \\
\hline$<50$ & $234(26.5)$ & 52.7 & $*$ \\
\hline $50-59$ & $262(29.7)$ & 52.8 & 63 \\
\hline $60-69$ & $199(22.5)$ & 45.4 & 40 \\
\hline$\geq 70$ & $188(21.3)$ & 35.2 & 26 \\
\hline \multicolumn{4}{|l|}{ Race } \\
\hline White & $693(78.5)$ & 49.4 & 58 \\
\hline Black & $123(13.9)$ & 29.7 & 25 \\
\hline Unknown & $67(7.6)$ & 54.9 & 61 \\
\hline \multicolumn{4}{|l|}{$\mathrm{N}$ stage } \\
\hline N0 & $144(16.3)$ & 61.6 & $*$ \\
\hline N1 & $372(42.1)$ & 48.7 & 56 \\
\hline N2 & $138(15.6)$ & 48.5 & 49 \\
\hline N3 & $177(20.0)$ & 43.3 & 36 \\
\hline $\mathrm{N}_{\mathrm{X}}$ & $52(5.9)$ & 19.7 & 18 \\
\hline \multicolumn{4}{|l|}{ M stage } \\
\hline M0 & $572(64.8)$ & 60.4 & $*$ \\
\hline M1 & $311(35.2)$ & 24.0 & 21 \\
\hline \multicolumn{4}{|l|}{ TNM stage } \\
\hline III & $572(64.8)$ & 60.4 & $*$ \\
\hline
\end{tabular}


IV

Surgery

No surgery

Not MRM

MRM

Unknown

Radiation

No

Yes

Chemotherapy

No

Yes

Tumor size

$<2 \mathrm{~cm}$

$2-5 \mathrm{~cm}$

$>5 \mathrm{~cm}$

Diffuse

Unknown

Breast Subtype

Her2-/HR+

Triple Negative

Her2+/HR-

Her2+/HR+
311(35.2)

24.0

21

21

162(18.3)

352(39.9)

14(1.6)

32.5

29

$487(55.2)$

396(44.8)

41.2

54.2

54.2

60.5

34

$25.1-15$

$\begin{array}{lll}721(81.7) & 52.1 & 63\end{array}$

$\begin{array}{lll}721(81.7) & 52.1 & 63\end{array}$

$68(7.7)$

53.3

54.0

$186(21.1)$

257(29.1)

43.2

37

$179(20.3)$

40.3

29

193(21.9)

50.1

66

293(33.2)

47.0

56

200(22.7)

28.7

20

151(17.1)

52.0

63

159(18.0)

71.3

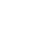

3


$\begin{array}{llll}\text { Unknown } & 80(9.1) & 39.6 & 22\end{array}$

ER

$\begin{array}{llll}\text { Negative } & 386(43.7) & 39.5 & 29 \\ \text { Positive } & 455(51.5) & 54.7 & * \\ \text { Unknown } & 42(4.8) & 35.8 & 21\end{array}$

PR

$\begin{array}{llll}\text { Negative } & 499(56.5) & 43.5 & 35 \\ \text { Positive } & 331(37.5) & 54.2 & 66 \\ \text { Other* } & 53(6.0) & 38.8 & 22\end{array}$

Her-2

$\begin{array}{llll}\text { Negative } & 496(56.2) & 39.3 & 35 \\ \text { Positive } & 311(35.2) & 62.7 & * \\ \text { Other* } & 76(8.6) & 40.2 & 26\end{array}$

Number of LN

$\begin{array}{llll}\text { Negative } & 104(11.8) & 73.0 & * \\ \text { Positive } & 422(47.8) & 52.8 & 63 \\ \text { Other } & 357(40.4) & 34.2 & 25\end{array}$

Marital status

$\begin{array}{llll}\text { Married } & 400(45.3) & 53.6 & 66\end{array}$

$\begin{array}{llll}\text { Divorce or widow } & \text { 238(27) } & 41.2 & 36\end{array}$

$\begin{array}{llll}\text { Single } & 201(22.8) & 45.3 & 39\end{array}$

$\begin{array}{llll}\text { Unknown } & 44(5.0) & 31.9 & 25\end{array}$

$2 *$ There are no results 